\title{
A Preliminary Numerical Simulation Study of Developing Ankle Foot Orthosis to Support Sit-To- Stand Movement in Children with Cerebral Palsy
}

\author{
Chihiro NAKAGAWA \\ Department of Mechanical \\ Engineering \\ Osaka Prefecture University \\ 1-1 Gakuen, Naka, Sakai, Osaka, \\ 599-8531, Japan
}

Shunsuke KUSADA

Department of Mechanical Engineering

Osaka Prefecture University

1-1 Gakuen, Naka, Sakai, Osaka, 599-8531, Japan

\author{
Ryo YONETSU \\ School of Comprehensive \\ Rehabilitation \\ Osaka Prefecture University \\ 3-7-30, Habikino, Habikino-shi, \\ Osaka, 583-8555, Japan
}

\author{
Tomohiro ITO \\ Department of Mechanical \\ Engineering \\ Osaka Prefecture University \\ 1-1 Gakuen, Naka, Sakai, Osaka, \\ 599-8531, Japan
}

\author{
Atsuhiko SHINTANI \\ Department of Mechanical Engineering \\ Osaka Prefecture University \\ 1-1 Gakuen, Naka, Sakai, Osaka, 599-8531, Japan
}

\begin{abstract}
The purpose of this study is to identify an effective method of support for the standing-up motion of children with cerebral palsy (CP). Experiments revealed remarkable differences in the shank and upper-body motions of children with $\mathrm{CP}$ compared with normally developed (ND) children. Shank tilt angles of $\mathrm{CP}$ children were smaller and their upper-body tilt angles were larger than those of ND children. The large upper-body tilt compensates for the smaller shank tilt but will cause back pain and/or deformation of the hip joint as they grow. It is therefore imperative to find a method of support to help CP children realize more natural motions (similar to those of ND children) to prevent these problems. The standing-up motion of ND children was adopted as the goal. Experiments identified a similarity in the angular variation between ND children's upper bodies and shanks; the standing-up motion of children with $\mathrm{CP}$ under that condition was then simulated using a two-dimensional four-link model of the human body. As a result of the numerical simulation, shank angles of $\mathrm{CP}$ children increased and their upper-body angles decreased from those measured during the experiments, which indicates that the proposed method of support is qualitatively effective at allowing $\mathrm{CP}$ children to realize a more natural standing-up motion.
\end{abstract}

Keywords-Cerebral; palsy; Standing-up motion; Motion analysis; Numerical simulation; Rigid link model

\section{INTRODUCTION}

Cerebral palsy (CP) is a chronic neurologic disorder caused by a static lesion in the immature brain and is characterized by deficits in movements and postural control. Thus, motor developments of $\mathrm{CP}$ have been more overdue or retarded than that of normally developing children [1]. Specially, CP resulted in difficulty in anti-gravity motor developments as in sit-to-stand (STS) movement because this movement requires adequate balance control between upper body and lower limbs, while the base of the support changes from a relatively larger area to a smaller area supported by the feet $[2,3]$. CP often observed excessive ankle plantar flexion during STS movement and had difficulty in shifting the body mass over their feet. In these ways, STS movement in children with CP was accomplished by various abnormal compensatory patterns, which include excessive trunk forward inclination and abrupt knee extension [4, 5]. Therefore, improving STS movement is an important rehabilitation goal for children with $\mathrm{CP}$.

Ankle foot orthosis (AFO) are frequently prescribed to correct skeletal malalignment in $\mathrm{CP}$ and has been used improving CP motor function in rehabilitation. However, most studies on effectiveness of AFO have focused on gait [6-9], the effectiveness of AFO on STS movement has limited [10, 11]. Compared with STS movement with barefoot, STS movement by using AFO was characterized by increasing ankle dorsiflexion. However, AFO did not change other proximal compensatory patterns of increased trunk forward titling and hip flexion [11]. This finding suggests that conventional AFO could not support STS movement fully in terms of coordinated motion between upper body and lower limbs. In other words, a new AFO which could promote better coordination between upper body and lower limbs would be needed.

In order to solve this issue, this preliminary study tried to construct a human model consists of 4 rigid bodies. From this model, the equations of motion are obtained. The studies on multi-body system are widely carried out especially in the collaborative control system between human movement and mechanical technology $[12,13]$. Therefore, the primary purpose of this study was to assess the kinetic characteristics of STS movement in children with CP by using 3 dimensional motion analysis system. Then, this working gives us a center of mass trajectory during STS to find a suitable control system design in AFO device. Moreover, the secondary purpose of this study was to clarify how our designed new AFO changes STS movement in children with $\mathrm{CP}$ by numerical simulations. These findings would help us conduct new AFO device in order to perform STS movement coordinately. 


\section{EXPERIMENT ON STANDING-UP MOTION}

\section{A. Experimental method}

The subjects for the experiment were two children with $\mathrm{CP}$ and two ND children; each child was 5 years old. The joint angles were measured while the subjects completed a standingup motion from a chair. The height of the chair was adjusted based on the subjects' knee positions, such that the knee and hip joints were orthogonal for each. The subjects were instructed to keep their upper bodies as straight as possible and to cross their arms in front of their chest to prevent their upper body from influencing the entire motion.

Using the motion analysis device Kinema Tracer of KISSEI COMTEC, ten markers were put on the subject's acromion, great trochanter, knee, malleolus, and fifth metatarsal head both sides of their body, as shown in Fig. 1. The motion was recorded by four video cameras and each joint angle $\theta_{i}(i=1,2,3,4)$ was measured by tracking the markers. We found that the angles of the lower body joints were dependent upon those of the upper body. In order to define the angles independently in the simulation, Eqs. (1)-(4) were used (see below). Each angle $\theta_{i}$ measured in the experiment was translated to angle $\phi_{i}$ ( $i$ $=1,2,3,4)$, defined from the horizontal axis shown in Fig. 2 .

$$
\begin{aligned}
& \phi_{1}=270^{\circ}-\theta_{1}+\theta_{2}-\theta_{2}-\theta_{4} \\
& \phi_{2}=90^{\circ}+\theta_{2}-\theta_{3}-\theta_{4} \\
& \phi_{3}=270^{\circ}-\theta_{3}-\theta_{4} \\
& \phi_{4}=90^{\circ}-\theta_{4}
\end{aligned}
$$

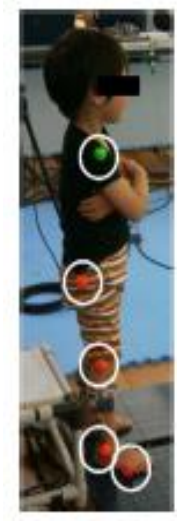

(a)

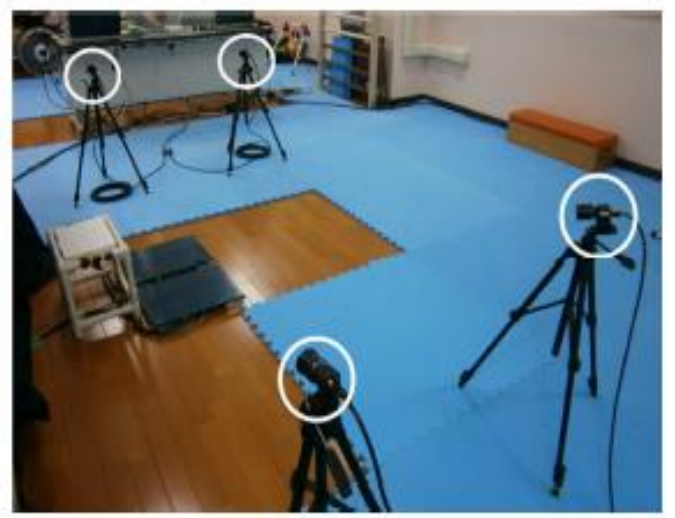

(b)
Fig. 1. The 3-dimensional motion analysis system. Positions of the markers are shown in (a). Video cameras were set in the experimental room

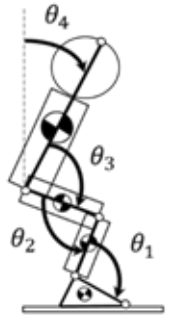

(a)

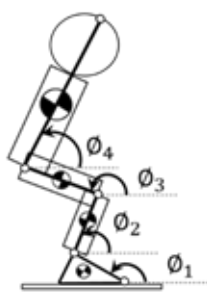

(b)
Fig. 2. The angular definition in the experiment is shown in (a) and the angular definition in the simulation is shown in (b)
This research was conducted after the Osaka Prefecture University Research Ethics Committee had approved the study. The purpose of this study was explained to the participants' parents both orally and in writing, and written consent was obtained.

\section{B. Experimental results}

Figure 3 shows the measurement results of each angle $\phi_{i}$ derived from Eqs. (1)-(4). The foot and thigh angle variations for both CP and ND children had similar tendencies, and differences between the two groups were not confirmed. The shank angle variations for the $\mathrm{CP}$ children were less than those of the ND children; Table 1 shows the initial and maximum tilt angles of the shank. The average maximum tilt angle for the ND subjects was about $10.5^{\circ}$; the maximum tilt angles for the two CP subjects were $4.31^{\circ}$ and $8.13^{\circ}$, respectively. We believe that these lower tilt angles for CP children are the result of spasticity making ankle dorsiflexion difficult. Meanwhile, the upper-body angle variations for the $\mathrm{CP}$ children were larger than those for the ND children; Table 2 shows the initial and maximum tilt angles of the upper body. The average maximum tilt angle for the ND subjects was about $32.5^{\circ}$; the maximum tilt angles for the two CP subjects were $46.62^{\circ}$ and $37.60^{\circ}$, respectively.

TABLE. I. INITIAL AND PEAK ANGLES OF THE SHANK

\begin{tabular}{l|l|l|l}
\hline \hline Subject & Initial $^{\circ}$ & Peak ${ }^{\circ}$ & Max. tilt $/{ }^{\circ}$ \\
\hline A (CP) & 89.83 & 85.52 & 4.31 \\
\hline B (CP) & 86.54 & 78.41 & 8.13 \\
\hline C (ND) & 79.24 & 68.75 & 10.49 \\
\hline D (ND) & 80.04 & 69.43 & 10.61 \\
\hline
\end{tabular}

TABLE. II. INITIAL AND PEAK ANGLES OF THE UPPER BODY

\begin{tabular}{l|l|l|l}
\hline \hline Subject & Initial $^{\circ}$ & Peak $^{\circ}$ & Max. tilt $/{ }^{\circ}$ \\
\hline A (CP) & 83.84 & 37.22 & 46.62 \\
\hline B (CP) & 81.74 & 44.14 & 37.60 \\
\hline C (ND) & 85.52 & 53.48 & 32.04 \\
\hline D (ND) & 85.75 & 52.75 & 32.97
\end{tabular}

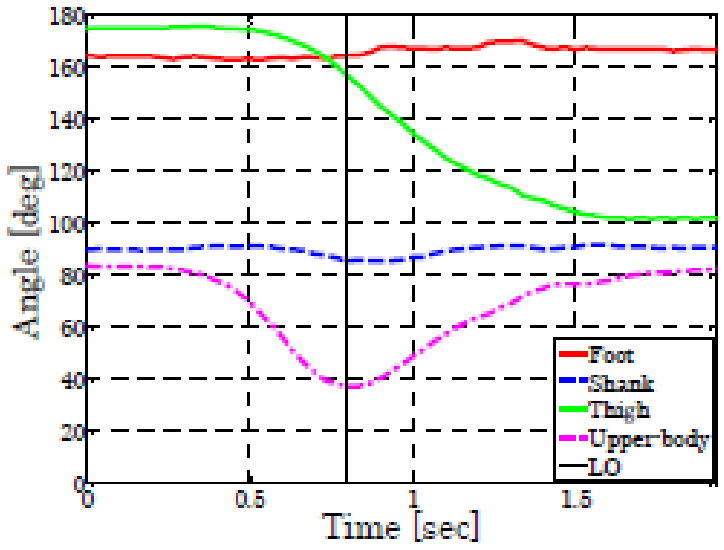

(a) Subject $\mathrm{A}(\mathrm{CP})$ 


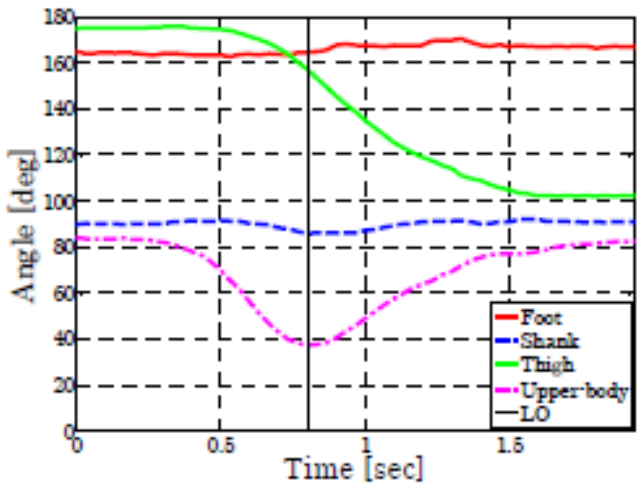

(b) Subject B (CP)

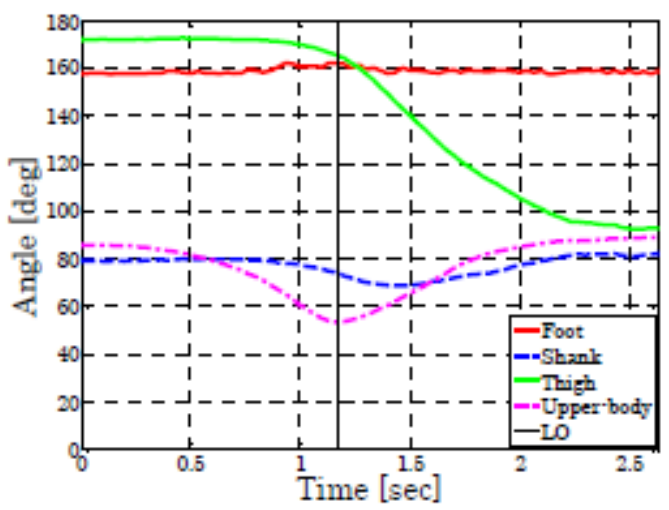

(c) Subject C (ND)

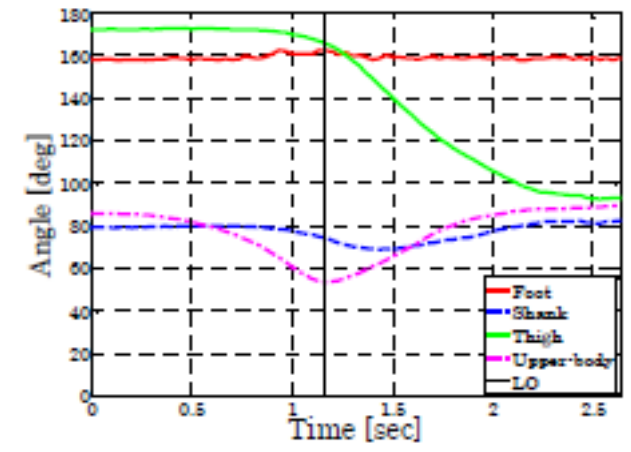

(d) Subject D (ND)

Fig. 3. Angular variations of CP subjects A and B and ND subjects C and D during the standing-up motion. The lines show the angular variations of foot (red), shank (blue), thigh (green), and upper-body (purple); the vertical black dashed line shows the point in time when the subject lifted their hips from the chair (LO)

\section{Discussion}

These results show that $\mathrm{CP}$ children have less shank tilt and more upper-body tilt than ND children during the standing-up motion. Figure 4 shows the correlation diagram of the maximum tilt angles of the shank and upper body; the two have a strong negative correlation. Therefore, it is assumed that the large angle of the upper body plays a considerable role in the smaller tilt angle of the shank, due to the spasticity, during the standing-up motion. The center of gravity $(\mathrm{COG})\left(x_{G}, y_{G}\right)$ for each subject during the standing-up motion is then defined using a twodimensional four-link rigid model, shown in Fig. 5. The definitions of each symbol are shown in Table 3 and the index $i$ indicates 1: foot, 2: shank, 3: thigh, and 4: upper-body, respectively. The position of the COG for the whole body is defined as Eqs. (5) and (6) using the position of the COG for each body part $\left(x_{i G}, y_{i G}\right)$.

$$
\begin{aligned}
& x_{G}=\frac{m_{1} x_{1 G}+m_{2} x_{2 G}+m_{3} x_{3 G}+m_{4} x_{4 G}}{m_{1}+m_{2}+m_{3}+m_{4}} \\
& y_{G}=\frac{m_{1} y_{1 G}+m_{2} y_{2 G}+m_{3} y_{3 G}+m_{4} y_{4 G}}{m_{1}+m_{2}+m_{3}+m_{4}}
\end{aligned}
$$

Figure 6 shows the trajectory of the COG of each subject as derived by Eqs. (5) and (6). The initial position is matched. The horizontal axis expresses the variation of the position of the COG for the horizontal direction and the vertical axis expresses that for the vertical direction. The position of the COG transits from the lower left to the upper right. From Fig. 6, we find that the trajectories of the position of the COG for CP children move forward along the horizontal direction more than those for ND children; however, the tendencies are almost similar. From this, we assume that $\mathrm{CP}$ children realize a similar transition of the COG as that attained by ND children by tilting their upper bodies excessively during the standing-up motion. This type of movement, used habitually to achieve functional motor skills when a normal movement pattern is unavailable, is called "compensatory movement." It has been suggested that-when there is instability during the standing-up motion-people tilt their upper bodies heavily, placing the COG near the seat (which is the supporting surface) and then standing up under the dynamically stabilized condition (Hirai et al., 2011). Because these motions cause back pain and hip joint deformation (Japanese Association of Rehabilitation Medicine, 2014), suppressing this excessive tilt in the upper body is desirable.

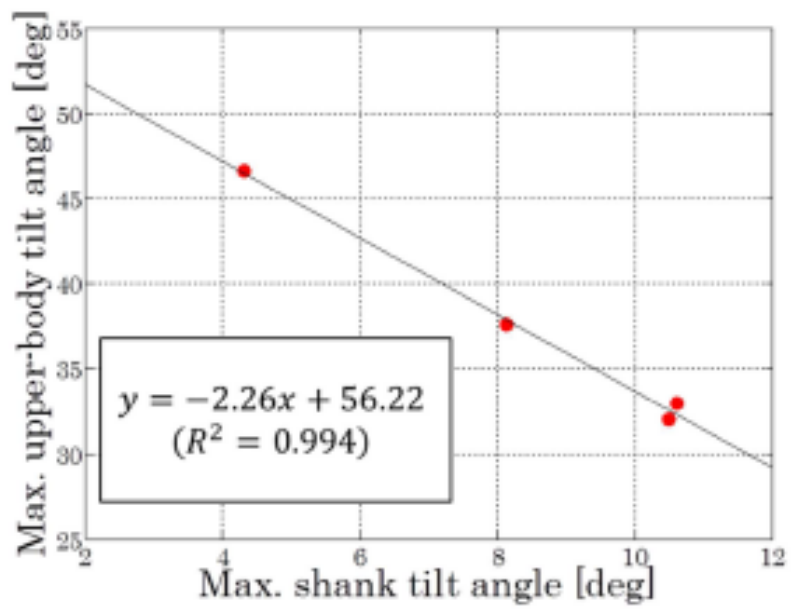

Fig. 4. Correlation between the max. shank tilt angle and max. upper-body tilt angle 


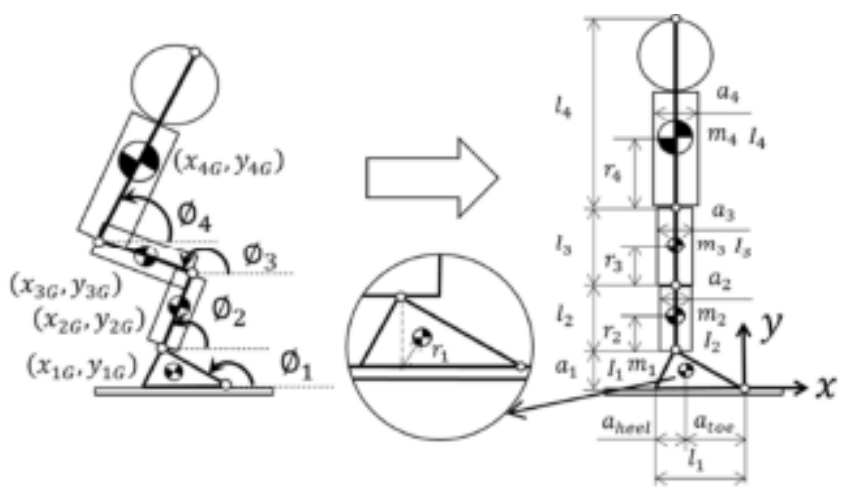

Fig. 5. Definition of the analytical model

TABLE. III. DEFINITIONS OF SyMBOLS IN THE ANALYTICAL MODEL

\begin{tabular}{c|c}
\hline \hline Symbol & Definition \\
\hline$m_{i}$ & Length of each part $i$ \\
\hline$l_{i}$ & Length of center of gravity of each part $i$ \\
\hline$r_{i}$ & Heel side of foot length \\
\hline$h_{\text {heel }}$ & Toe side of foot length \\
\hline$h_{\text {toe }}$ & Thickness of each part $i$ \\
\hline$a_{i}$ & Inertia moment of each part $i$ \\
\hline$I_{i}$ & Length from waist to top of head \\
\hline$l_{4}$ & Center of gravity (COG) of each part $i$ \\
\hline$\left(x_{i g}, y_{i g}\right)$ &
\end{tabular}

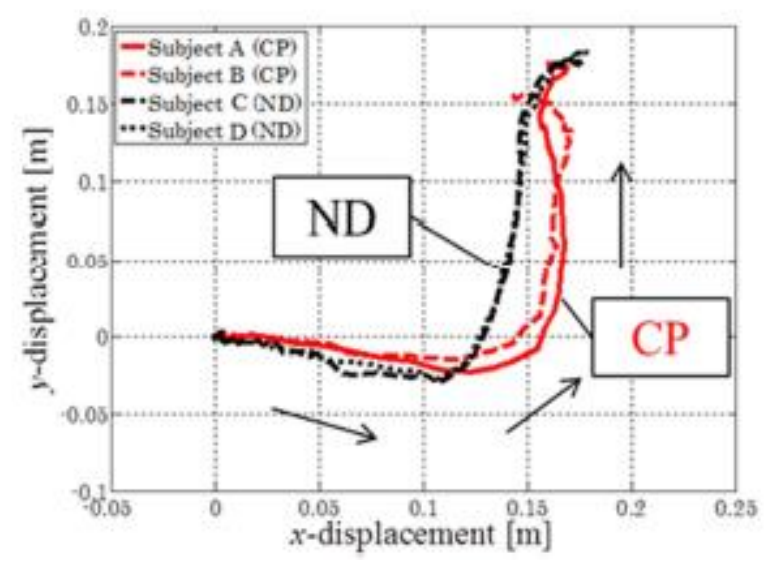

Fig. 6. COG trajectories during the stand-up motion. Red solid and dashed lines show CP subjects A and B; black solid and dashed lines show ND subjects $\mathrm{C}$ and $\mathrm{D}$

\section{NUMERICAL SIMULATIONS}

\section{A. Standing-up motion of ND children}

The experiment detailed above shows the problem caused by excessive upper-body tilt in children with CP. Now, the numerical simulations detailed in this section analyze a supporting method designed to allow children with CP to move similarly to ND children in order to prevent the problem caused by excessive upper-body tilt. To do so, we use measurement results from the experiment to clarify the characteristics of ND children's standing-up motions.

Figure 7 shows the angles $\theta_{i}(i=1,2,3,4)$ of each body part of ND children based on the angle definition shown in Fig. 2(a). We find that the tendencies of the knee joint angle $\theta_{2}$ and hip joint angle $\theta_{3}$ correspond after the hip is lifted. Therefore, $\theta_{2}=$ $\theta_{3}$ is one characteristic of ND children. This tendency was also confirmed by previous experiments of the standing-up motion in which nine healthy adults (six men and three women) with an average age of 27 years (Doorenbosch et al., 1994) and 47 healthy adults (27 men and 20 women) with an average age of 20.1 years (Tully et al., 2005) were used as subjects. This condition is shown as

$$
\theta_{2}=\theta_{3}
$$

By applying the conversion equations shown in Eqs. (1)-(4), following equations are obtained:

$$
\begin{aligned}
& \theta_{2}=180^{\circ}+\phi_{2}-\phi_{3} \\
& \theta_{3}=180^{\circ}-\phi_{3}+\phi_{4}
\end{aligned}
$$

By substituting Eqs. (8) and (9) into Eq. (7), the following condition is obtained:

$$
\phi_{2}=\phi_{4}
$$

which means that the lower-leg angle $\phi_{2}$ and upper-body angle $\phi_{4}$ correspond during the standing-up motion. In this study, Eq. (10) is defined as the condition of ND children's standing-up motion.

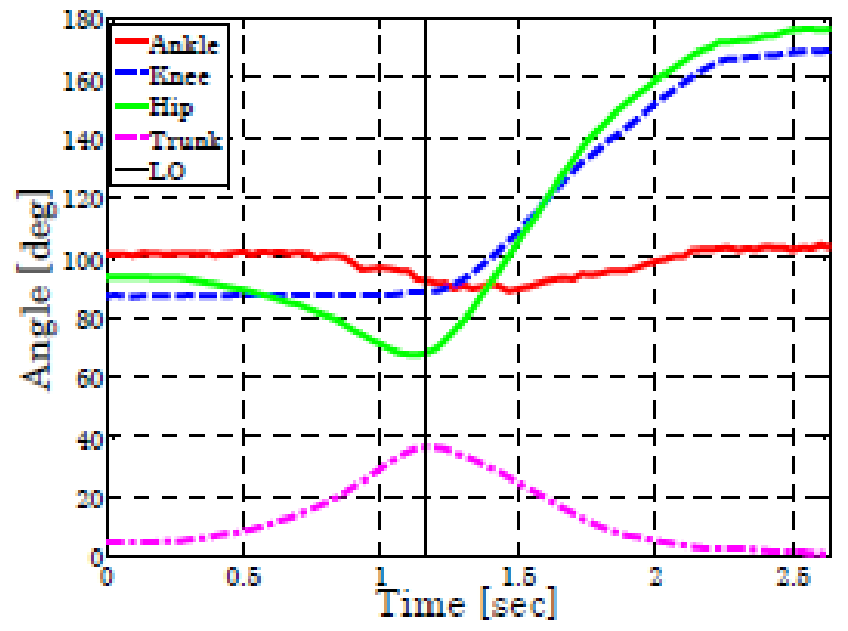

(a) Subject C (ND) 


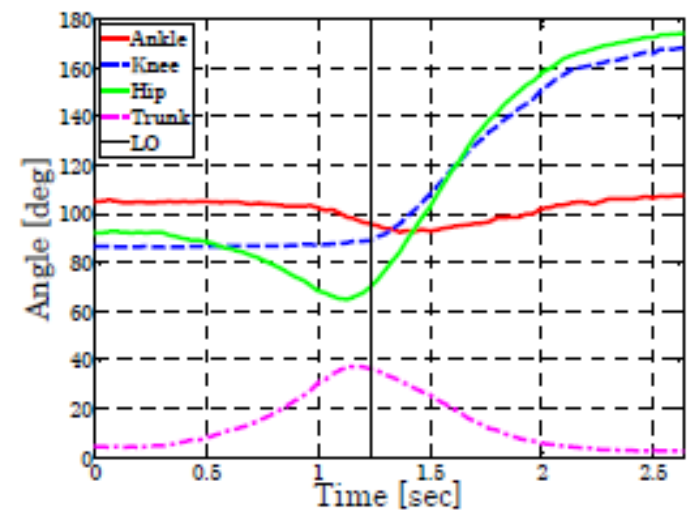

(b) Subject D (ND)

Fig. 7. Angles $(=1,2,3,4)$ of each body part of ND children

\section{B. Numerical simulations}

We have focused on the angles of each body part and on the position of the COG as factors that determine a person's standing-up motion. As shown in Eqs. (5) and (6), the COG's whole-body position is determined by the COG of each rigid body in the analysis model and the COG of each rigid body is determined by the angle of each body part. Therefore, when the COG's position is determined beforehand, the angle of each body part can be derived by inverse operation in the numerical analysis.

As mentioned earlier, there have been studies of AFOs that use actuators to support lower-leg motion. Using a similar idea, we investigate the influence of lower-leg angle changes on the motion of the upper-body angle. By numerically simulating the standing-up motion of CP children, we investigate the possibility of improving both the lack of lower-leg tilt and the excessive upper-body tilt that are characteristic of CP children. We assume that by improving these two characteristics, the standing-up motion of CP children will become more similar to the standingup motion of ND children. To do so, we use the following three methods:

\section{Method 1.}

Target the lower-leg angle of ND children.

\section{Method 2.}

Determine the lower-leg angle of $\mathrm{CP}$ children based on the standing-up motion of ND children.

\section{Method 3.}

Apply ND children's COG position to CP children.

\section{1) Targeting the lower-leg angle of $\mathrm{ND}$ children}

In this method, ND children's position of COG is regarded as equal to $\mathrm{CP}$ children's original position of COG. By inputting the lower-leg angle of ND children, CP children's lower-leg tilts are numerically simulated and upper-body angles derived. From Eq. (5), the definition of COG, the upper-body angle $\emptyset_{4}$ is derived as

$$
\phi_{4}=\cos ^{-1}\left(\frac{1}{r_{4}}\left\{\frac{\left(\sum_{i=1}^{4} m_{i}\right) x_{G}-\sum_{i=1}^{3} m_{i} x_{i G}}{m_{4}}-\sum_{i=1}^{3} l_{i} \cos \phi_{i}\right\}\right)
$$

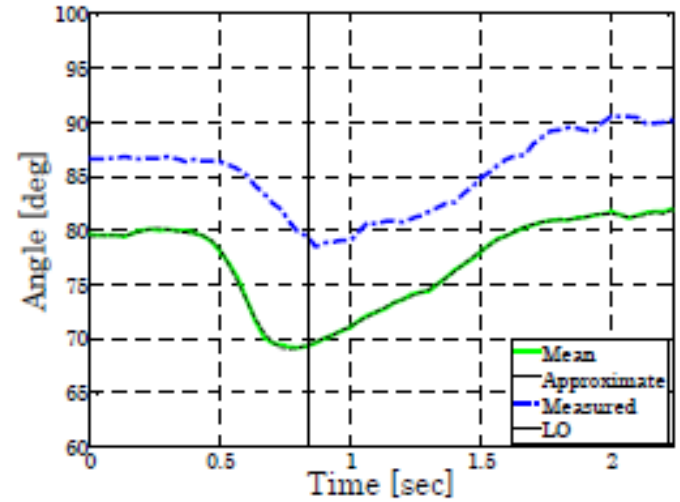

(a) Subject A (CP)

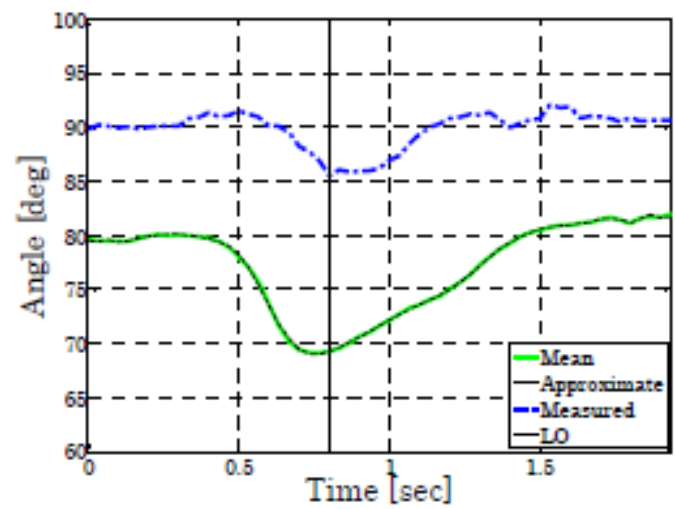

(b) Subject B (CP)

Fig. 8. Comparison of shank angular variations. Each line shows the mean angular variations between ND children (green), the approximate line of mean between ND children (black), and the measured angles of CP subjects A and B (blue)

The upper-body angle $\phi_{4}$ is derived by substituting the foot angle $\phi_{1}$, the thigh angle $\phi_{3}$, the CP children's COG position $x_{G}$, and the ND children's lower-leg angle. Figure 8 shows the measured values of two CP children's lower-leg angles and the average lower-leg angle of two ND children and its approximate curve. Figure 9 compares the CP children's measured upperbody angles with those derived from Eq. (11). Table 4 shows the maximum tilt angle from the initial condition. We see from Fig. 9 and Table 4 that the maximum tilt angle of the upper body decreases by $13.46^{\circ}$ for subject $A$ and $5.26^{\circ}$ for subject B. Thus, the upper body's excessive tilt is improved.

TABLE. IV. MAXIMUM MEASUREd UpPER-BOdy TILT ANGLes, ThOSE DERIVED FROM EQ. (11), AND THE DIFFERENCE BETWEEN THEM

\begin{tabular}{l|l|l|l}
\hline \hline Subject & Measured $/{ }^{\circ}$ & Derived $/{ }^{\circ}$ & Difference $^{\circ}$ \\
\hline A (CP) & 46.62 & 33.16 & 13.46 \\
\hline B (CP) & 37.60 & 31.98 & 5.26 \\
\hline
\end{tabular}




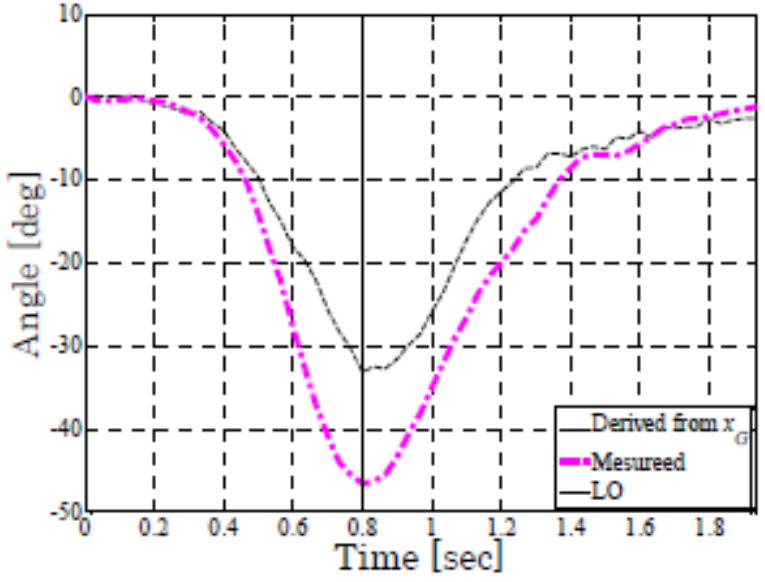

(a) Subject A (CP)

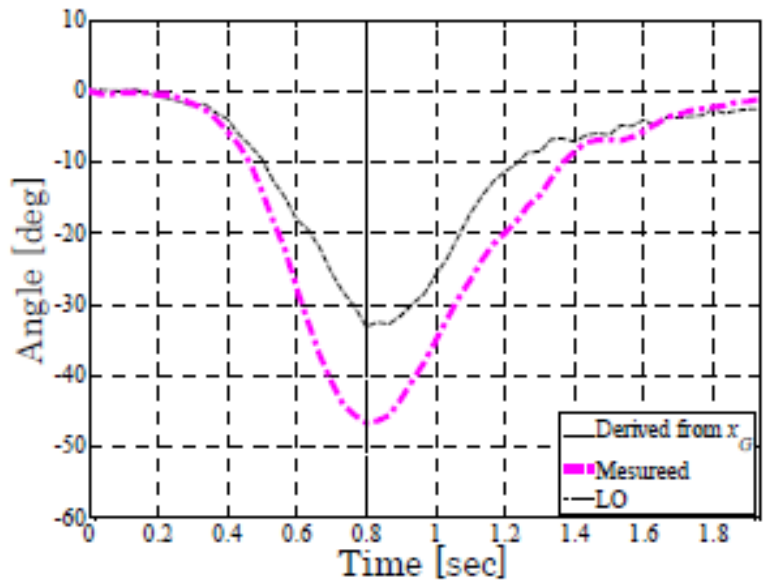

(b) Subject B (CP)

Fig. 9. Comparison of upper-body angular variations. Each line shows the angular variations derived by Eq. (11) (black) and measured (purple) of $\mathrm{CP}$ subjects A and B

2) Determining the lower-leg angle based on the standing-up motion of ND children

In this method, the position of the COG in CP children is regarded as equal to the original motion of $\mathrm{CP}$ children. The upper-body angle is derived from Eq. (11), regarding the average of the lower-leg angle and upper-body angle as the lower-leg angle, based on the condition of ND children's standing-up motion. The angle of the lower leg is defined as

$$
\phi_{24}=\frac{\phi_{2}+\phi_{4}}{2}
$$

Figure 10 shows the mean of the lower-leg and upper-body angles $\phi_{24}$. We see that the mean angle $\phi_{24}$ is larger than the lower-leg angle $\phi_{2}$ measured in the experiment. Thus, using $\phi_{24}$ as the lower-leg angle will improve the excessive upperbody tilt. Figure 11 shows the upper-body angle $\phi_{4}$ derived by substituting $\phi_{24}$ into the lower-leg angle $\phi_{2}$ in Eq. (11).

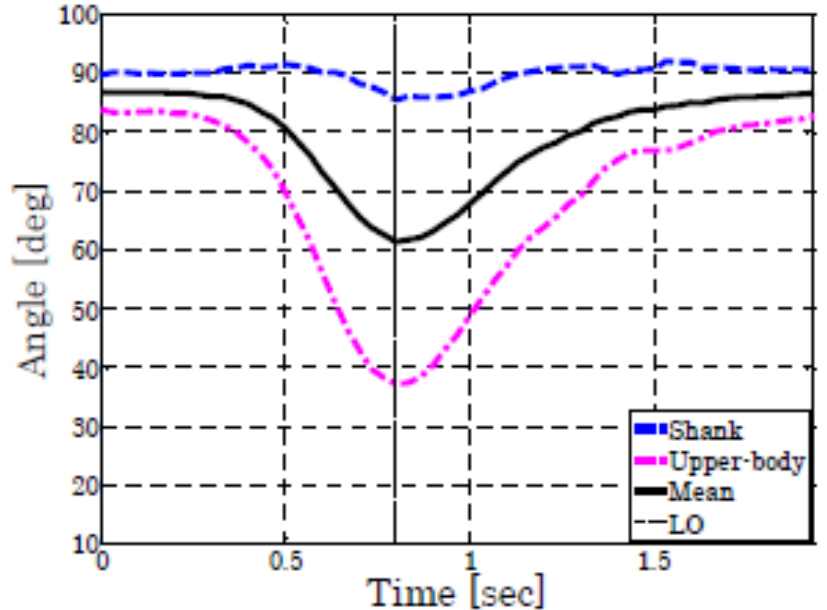

(a) Subject A (CP)

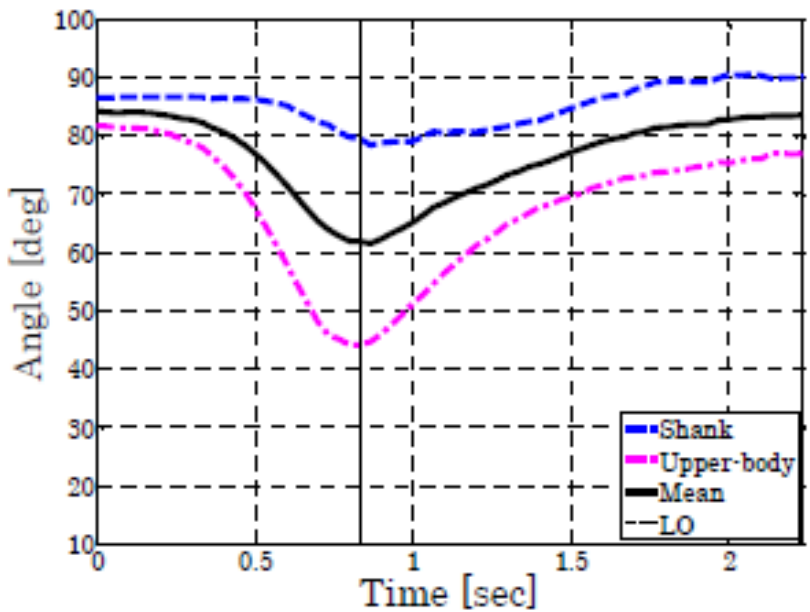

(b) Subject B (CP)

Fig. 10. Comparison of angular variations. Each line shows the angular variations of the measured shank (blue), the measured upper body (purple), and the mean between the shank and upper body (black) of CP subjects A and B

Table 5 shows the maximum tilt angle from the initial posture. From Fig. 11 and Table 5, we see that the maximum upper-body angle decreases. However, the maximum tilt angles of ND children were about $32^{\circ}$, so the angles resulting from the simulation (13.75 and $\left.15.44^{\circ}\right)$ are too small. Therefore, the excessive upper-body tilt was improved but is still different from the standing-up motion of ND children.

TABLE. V. MAXIMUM UPPER-BODY TILT ANGLE MEASURED IN THE EXPERIMENT AND DERIVED USING THE MEAN ANGLE BETWEEN THE MEASURED SHANK AND UPPER-BODY ANGLES, AND THE DIFFERENCE BETWEEN THEM

\begin{tabular}{l|l|l|l}
\hline Subject & Measured $/{ }^{\circ}$ & Derived $/{ }^{\circ}$ & Difference $^{\circ}$ \\
\hline $\mathrm{A}(\mathrm{CP})$ & 46.62 & 13.75 & 32.87 \\
\hline $\mathrm{B}(\mathrm{CP})$ & 37.60 & 15.44 & 22.16 \\
\hline
\end{tabular}




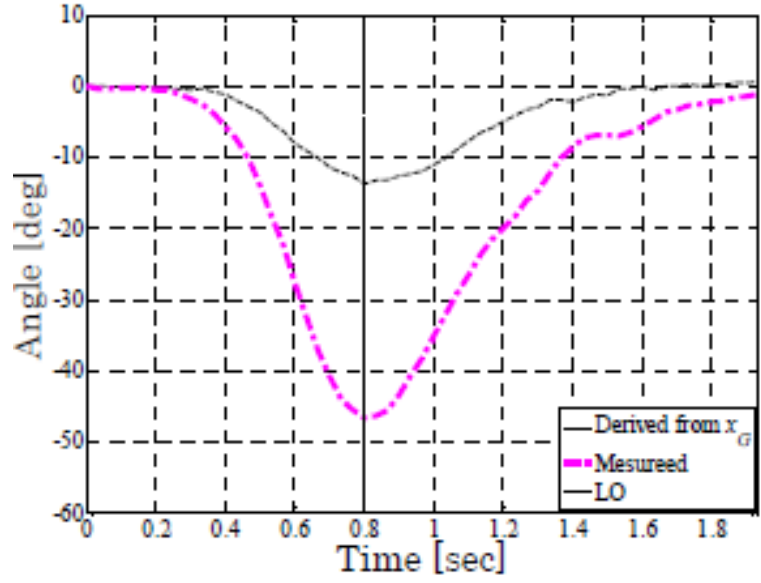

(a) Subject A (CP)

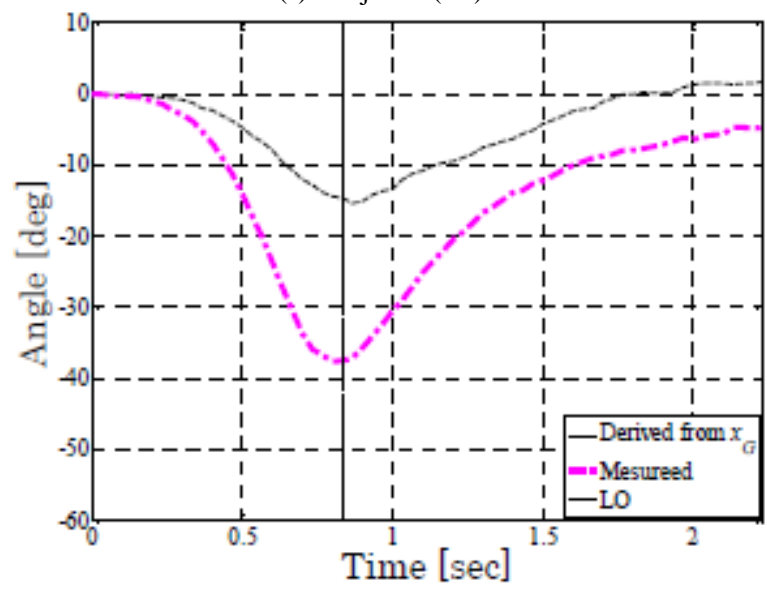

(b) Subject B (CP)

Fig. 11. Comparison of angular variations. Each line shows the angular variations derived by Eq. (11) (black) and the measured upper-body angles (purple) of CP subjects A and B

\section{3) Applying ND children's COG position}

In Section 3.1, conditional Eq. (11) was defined using the measurement data of ND children as a reference. Assuming that lower-leg angle $\phi_{2}$ and upper-body angle $\phi_{4}$ correspond, then angle $\phi_{24}$ is derived using the equation $\phi_{2}=\phi_{4}=\phi_{24}$ and the definitional equation of the position of the COG, found in Eq. (5).

$$
\begin{aligned}
& \phi_{24}=\cos ^{-1} \\
& {\left[\left\{\frac{\left(\sum_{i=1}^{4} m_{i}\right) x_{G}-\left(m_{2} r_{1}+\sum_{i=2}^{4} m_{i} l_{2}\right) \cos \phi_{1}}{m_{2} r_{2}+m_{3} l_{2}+m_{4} l_{2}+m_{4} r_{4}}\right\}\right.} \\
& \left.-\left\{\frac{\left(m_{3} r_{3}+m_{4} l_{3}\right) \cos \phi_{3}}{m_{2} r_{2}+m_{3} l_{2}+m_{4} l_{2}+m_{4} r_{4}}\right\}\right]
\end{aligned}
$$

We substitute the approximate curve of ND children's COG into Eq. (13) and investigate the effect that has on bringing the motions of CP children closer to those of ND children. Figure 12 shows the derived lower-leg and upper-leg angle $\phi_{24}$. Table 6 shows the maximum tilt angle from the initial posture.

From Fig. 12 and Table 6, we find that the maximum tilt angle of the lower leg increases while that of the upper body decreases. Table 6 shows that the measured value of ND children's maximum lower-leg tilt angle is about $10.5^{\circ}$ and that of the upper body is about $32^{\circ}$; however, the derived lower-leg angle in this simulation is larger (to a certain degree), while the derived upper-body angle is much smaller. Therefore, the increased lower-leg tilt and reduced upper-body tilt are improved although the motions of $\mathrm{CP}$ children are still different from those of ND children.

TABLE. VI. THE MAXIMUM SHANK AND UPPER-BODY TILT ANGLES MEASURED IN THE EXPERIMENT AND DERIVED BY EQ. (13)

\begin{tabular}{l|l|l|l}
\hline \hline Subject & Measured shank $/{ }^{\circ}$ & $\begin{array}{l}\text { Measured upper-body } \\
{ }^{\circ}\end{array}$ & Derived ${ }^{\circ}$ \\
\hline A (CP) & 46.62 & 4.31 & 14.34 \\
\hline B (CP) & 37.60 & 8.13 & 13.50 \\
\hline
\end{tabular}

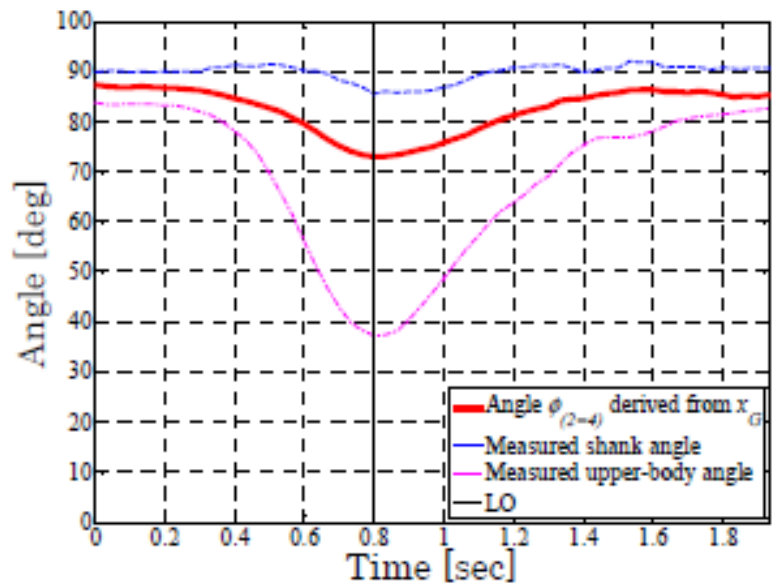

(a) Subject A (CP)

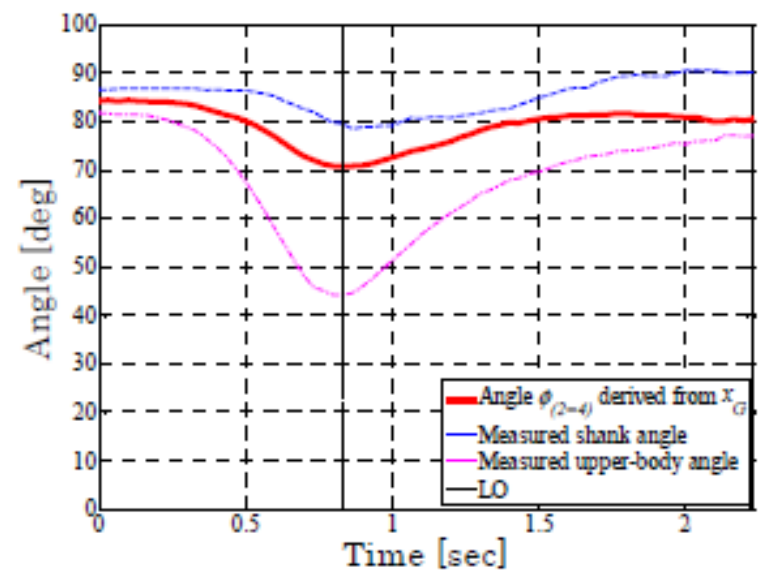

(b) Subject B (CP)

Fig. 12. Comparison of angular variations. Each line shows the angular variations of the angles derived by Eq. (13) (red), the measured shank (blue), and the measured upper-body (purple) of CP subjects A and B

\section{Discussion of the simulation result}

Because the measured lower-leg angles of ND children are directly substituted for $\mathrm{CP}$ children in the first method (Section 3.2.1), it makes sense that those simulation results most closely resemble the behavior of ND children. We therefore conclude 
that AFO support for CP children will be most effective when the lower-leg angles of ND children are taken into consideration.

On the other hand, we also tried to reproduce the behavior of ND children using characteristics of their standing-up motion (Sections 3.2.2 and 3.2.3). In both cases, the numerical simulations confirm improvements in lower-leg and upper-body tilt; however, compared with the actual behavior of ND children, the simulation results show larger lower-leg tilt and less upperbody tilt. We believe this is caused by our not setting a threshold for all angles. By calculating the COG position in the numerical simulation using the substituted lower-leg angle and by setting a threshold tilt angle that avoids the risk of falling down, we believe that behavior closer to that of ND children can be achieved, as can AFO application.

Thus, the simulation results show improvement in the evident characteristics of $\mathrm{CP}$ children during their standing-up motion and the possibility of obtaining $\mathrm{CP}$ behavior that is nearer that of ND children in a qualitative manner.

\section{CONCLUSIONS}

Supportive methods for obtaining standing-up motions in $\mathrm{CP}$ children that are similar to those of ND children have been investigated with the assumption that AFOs use actuators to support ankle dorsiflexion. Through numerical simulations, we found that it is qualitatively possible to achieve $\mathrm{CP}$ standing-up behavior that is near to that of ND children by applying certain characteristics of ND children's behavior, specifically, that the lower-leg angle corresponds to the upper-body angle. The experiments using the proposed methods will be the future works.

\section{ACKNOWLEDGMENT}

The part of study is supported by Adaptable and Seamless Technology transfer Program through target-driven R\&D (A-
STEP) Grant (AS242Z01706K). We appreciate the support of Japan Science and Technology Agency.

\section{REFERENCES}

[1] Milani-Comparetti A, Gidoni AE. "Pattern analysis of motor development and its disorders," Dev Med child Neurol 9, pp.625-630, 1967.

[2] Riley PO, Schneckman ML, Mann RW, Hodge WA. "Mechanics of a constrained chair-rise," J Biomech, 24, pp. 77-85, 1991.

[3] Schultz AB, Alexander NB, Ashton-Miller JA. "Biomechanical analyses of rising from a chair". J Biomech, 25, pp.1383-1391, 1992.

[4] Park ES, Park CI, Lee HJ, Kim DY, Lee DS, Cho SR. "The characteristics of sit-to-stand transfer in young children with spastic cerebral palsy based on kinematic and kinetic data", Gait Posture, 17, pp.43-49, 2003.

[5] Yonetsu R, Iwata A, Surya J, Unase K, Shimizu J. "Sit-to-stand movement changes in preschool-aged children with spastic diplegia following one neurodevelopmental treatment session- a pilot study" Disabil Rehabil, 37, pp.1643-1650, 2015.

[6] Radtka SA, Skinner SR, Dixon DM, et al. "A comparison of gait with solid, dynamic, and no ankle-foot orthoses in children with spastic cerebral palsy", Phys Ther, 77, pp.395-409, 1997.

[7] Crenshaw S, Herzog R, Castagno P, et al. "The efficacy of tone-reducing features in orthotics on the gait of children with spastic diplegic cerebral palsy", J Pediatr Orthop, 20, pp.210-216, 2000.

[8] Desllovere K, Molenaers G, Gestel LV, et al. "How can push-off be preserved during use of an ankle foot orthosis in children with hemiplegia? A prospective controlled study", Gait Posture, 24, pp.142-151, 2006.

[9] Van Gestel L, Molenaers G, Huenaerts C, et al. "Effect of dynamic orthoses on gait: a retrospective control study in children with hemiplegia", Dev Med Child Neurol, 50, pp.63-67, 2008.

[10] Wilson H, Harideri N, Song K, Telford D. "Ankle-foot orthoses for preambulatory children with spastic diplegia”, J Pediatr Orthop, 17, pp.370-376, 1997.

[11] Park ES, Park CI, Chang HJ, Choi JE, Lee DS. "The effect of hinged ankle-foot orthoses on sit-to-stand transfer in children with spastic cerebral palsy”, Arch Phys Med Rehabil, 85, pp.2053-2057, 2004.

[12] Nakagawa C, Morita Y. Shintani A, Ito T., "Standing posture analysis of a human on a four-wheel stand-up-type personal mobility vehicle", Transaction of the JSME (in Japanese), 82: No,838: 1-13, 2016.

[13] Kim JH, Xiang Y, Yang J, Arora JS, Abdel-Malek K, "Dynamic motion planning of overarm throw for a biped human multibody system", Multibody Syst Dyn, 24, pp.1-24, 2010. 